\title{
Delayed umbilical cord separation in alloimmune neutropenia
}

\author{
A S Kemp, L Lubitz
}

\begin{abstract}
Delayed umbilical cord separation in association with neonatal alloimmune neutropenia is reported. Delayed umbilical cord separation has been described in association with defects in neutrophil function. The present case indicates that deficiency in neutrophil number should also be considered as a cause of delayed cord separation.
\end{abstract}

(Arch Dis Child 1993;68:52-3)

The factors contributing to umbilical cord separation are not fully understood. It is, however, considered likely that this is mediated through migration of neutrophils with a subsequent inflammatory response which results in digestion of the umbilical cord. Major support for this hypothesis comes from the observation of delayed umbilical cord separation in subjects with a deficiency of leucocyte adherence related glycoproteins in which cell migration from the vascular compartment is severely impaired. ${ }^{1} 2$ In this condition there is a marked deficiency of neutrophil infiltration into the cord. If the above hypothesis is correct, in addition to defects in neutrophil migration, it is possible that delayed cord separation may be associated with neutropenia.

\section{Case report}

A boy was born by caesarean section at 36 weeks' gestation, birth weight 2190 g. A blood film on day 2 showed no neutrophils and a similar finding was observed on day 5 when he developed symptoms and radiological features of necrotising enterocolitis that was managed conservatively and settled over the next nine days. Because of the total absence of neutrophils he was given intravenous gammaglobulin $9 \mathrm{~g}$ over days 5 to 7 , a further $2 \mathrm{~g}$ on day 13 , and $6 \mathrm{~g}$ on day 16 . His neutrophil count gradually improved: $0.11 \times 10^{9} / 1$ on day $7,0.12 \times 10^{9} / 1$ on day 8 , $0.56 \times 10^{9} / 1$ on day $15,0.86 \times 10^{9} / 1$ on day 18 , and $5 \cdot 4 \times 10^{9} / 1$ on day 20 (normal $1-9 \times 10^{9} / 1$ ). He has not had subsequent neutropenia and his umbilical cord did not become infected and separated on day 55 .

Studies performed on mother's and father's serum and cells collected on day 4 showed that the mother's neutrophils were homozygous for the neutrophil specific antigen NA1, the father was NA1 NA2 and the mother had a strong anti-NA2 antibody in her serum.
Serum was not collected from the infant at this time. Neutrophils collected from the infant after return to normal of the neutrophil count were NA1 NA2. Maternal sera showed an extremely high titre of agglutinating antibody directed against the infant's neutrophils. Neutrophil function studies on the infant showed normal nitroblue tetrazolium reduction, normal neutrophil chemotaxis, and normal expression of antigens reacting with the monoclonal antibody OKM1, indicating the presence of leukocyte cell adhesion molecules.

\section{Discussion}

The mean times of cord separation in two large studies were 7 days $^{3}$ and 14 days. ${ }^{4}$ The upper limit of separation was 29 days $^{3}$ and 45 days. ${ }^{4}$ The present case demonstrates markedly delayed umbilical cord separation that is clearly outside the limit of normal. While the mechanism of cord separation is not clear, several authors have suggested that an inflammatory response due to neutrophil infiltration is a significant factor. ${ }^{14}$ The association of a delay in cord separation with neonatal alloimmune neutropenia, which has resulted in the total absence of neutrophils from the circulation for the first five days of life, and eventual restoration to normal on day 20, suggests that the marked neutropenia has contributed to the delay in umbilical cord separation. Delayed cord separation has been observed in subjects with a deficiency of leukocyte adherence related glycoproteins. The histology of the cord in these cases has shown a marked deficiency of neutrophil infiltration. ${ }^{2}$ In this condition the ability of neutrophils to migrate from the vascular compartment is seriously impaired. A similar paucity of neutrophil infiltration would obviously be present in cases of profound neutropenia. This case provides further evidence that neutrophil infiltration is involved in cord separation and indicates that, in addition to defects of neutrophil function, neutropenia should also be considered in subjects with delayed umbilical cord separation.

The incidence of alloimmune neonatal neutropenia is unknown but some authorities have suggested it may affect up to $3 \%$ of live births. ${ }^{5}$ In the absence of an indication to perform a neutrophil count many milder cases may not be detected. ${ }^{6}$ If some degree of neonatal neutropenia is as common as has been suggested this may be one factor con- 
tributing to the normal variation in time of cord separation.

1 Hayward AR, Leonard J, Wood CBS, Harvey BAM, Greenwood MC, Soothill JF. Delayed separation of the umbilical cord, widespread infections, and defective neutrophil mobility. Lancet 1979;i:1099-101.

2 Anderson DC, Schmalsteig FC, Finegold MJ, et al. The severe and moderate phenotypes of heritable Mac-1, LFA-1 deficiency: their quantitative definition and rela-
tion to leukocyte dysfunction and clinical features. $\mathcal{f}$ Inf Dis 1985;152:668-89.
3 Oudesluys-Murphy AM, Eilers GAM, De Groot CJ. The time of separation of the umbilical cord. Eur $\mathcal{F}$ Pediatr 1987;146:387-9.

4 Novack AH, Mueller B, Ochs H. Umbilical cord separation in the normal newborn. Am 7 Dis Child 1988; 142:220-3.

5 Curnutte JT, Boxer CA. Disorder of granulopoiesis and granulocyte function. In: Nathan DG, Oski FA, eds. Hematology of infancy and childhood. Philadelphia: WB Saunders, 1987:797-847.

6 Minchinton RM, McGrath KM. Alloimmune neutropenia-a neglected diagnosis? Med $\mathcal{f}$ Aust 1987;147: nia-a neg- 